\title{
Anaphylaxis following quadrivalent human papillomavirus vaccination
}

\author{
Julia M.L. Brotherton MD MPH, Mike S. Gold MD, Andrew S. Kemp MD PhD, \\ Peter B. Mclntyre MD PhD, Margaret A. Burgess MD, Sue Campbell-Lloyd RN, \\ on behalf of the New South Wales Health HPV Adverse Events Panel
}

Published at www.cmaj.ca on Sept. 1, 2008.

$\infty$ See related commentary by Halsey, page 509

\section{ABSTRACT}

Background: In 2007, Australia implemented the National human papillomavirus (HPV) Vaccination Program, which provides quadrivalent HPV vaccine free to all women aged 12-26 years. Following notification of 7 presumptive cases of anaphylaxis in the state of New South Wales, Australia, we verified cases and compared the incidence of anaphylaxis following HPV vaccination to other vaccines in comparable settings.

Methods: We contacted all patients with suspected anaphylaxis and obtained detailed histories from telephone interviews and a review of medical records. A multidisciplinary team determined whether each suspected case met the standardized Brighton definition. Some participants also received skin-prick allergy testing for common antigens and components of the HPV vaccine.

Results: Of 12 suspected cases, 8 were classified as anaphylaxis. Of these, 4 participants had negative skin-prick test results for intradermal Gardasil. From the $269680 \mathrm{HPV}$ vaccine doses administered in schools, 7 cases of anaphylaxis were identified, which represents an incidence rate of 2.6 per 100000 doses $(95 \% \mathrm{Cl} 1.0-5.3$ per 100 000). In comparison, the rate of identified anaphylaxis was 0.1 per 100000 doses $(95 \% \mathrm{Cl} 0.003-0.7)$ for conjugated meningococcal C vaccination in a 2003 school-based program.

Interpretation: Based on the number of confirmed cases, the estimated rate of anaphylaxis following quadrivalent HPV vaccine was significantly higher than identified in comparable school-based delivery of other vaccines. However, overall rates were very low and managed appropriately with no serious sequelae.

Une version française de ce résumé est disponible à l'adresse www.cmaj.ca/cgi/content/full/179/6/525/DC1

CMAJ 2008;179(6):525-33

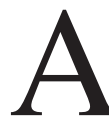
naphylaxis to a vaccine, or to a known ingredient, is widely recognized as the only absolute contraindication to vaccination. Vaccine ingredients with the potential to cause anaphylaxis in sensitized people include egg proteins (e.g., influenza vaccine), gelatin (in live viral vaccines) and antibiotics. ${ }^{1}$ Although anaphylaxis due to vac- cination is rare, with an estimated incidence of $0.1-1$ per 100000 doses, ${ }^{2}$ its occurrence, especially if death occurs, has the potential to severely damage public and provider confidence in vaccination.

Identified cases of anaphylaxis following vaccination tend to occur less than 1 hour after vaccination. ${ }^{1}$ Measurement of anaphylaxis and comparisons between different settings are made difficult, however, by the lack of a universally agreed definition of anaphylaxis ${ }^{3}$ and by variable presentation and rate of progression, especially if adrenaline is given. Rates of anaphylaxis reported by vaccine safety surveillance systems may be underreported (apart from anaphylactic shock) and vary depending upon the population vaccinated, the type of vaccines used and the type of surveillance system. As they are newly licensed, the rate of anaphylaxis that may occur following immunization with prophylactic human papillomavirus (HPV) vaccines is currently unknown and awaits results from vaccine safety surveillance systems.

In late June 2007, we identified a potential vaccine safety signal (reported information about a previously unknown or incompletely documented but possible causal adverse event following vaccination ${ }^{4}$ ), when 7 presumptive cases of anaphylaxis were reported following quadrivalent HPV vaccination. We aimed to estimate the rate of anaphylaxis following HPV vaccination.

\section{Methods}

\section{New South Wales HPV vaccination program}

In 2007, Australia implemented a fully funded program of human papillomavirus vaccination for all women aged 12-26 years, using 3 doses of quadrivalent HPV vaccine (Gardasil, Merck) that protects against HPV types 6, 11, 16 and 18. The vaccine was delivered through schools for those aged 12-18 years and primary care for those aged 19-26 years. The state of

From the National Centre for Immunisation Research and Surveillance of Vaccine Preventable Diseases (Brotherton, Mclntyre, Burgess), The Children's Hospital at Westmead, and the University of Sydney, New South Wales; the Department of Paediatrics (Gold), Women's and Children's Hospital, Adelaide, South Australia; Department of Allergy and Immunology (Kemp), The Children's Hospital at Westmead; Discipline of Paediatrics and Child Health (Kemp, Burgess), University of Sydney, New South Wales; and the New South Wales Department of Health (Campbell-Lloyd), North Sydney, New South Wales, Australia 


\section{Box 1: Brighton case definition* of anaphylaxist}

For all levels of diagnostic certainty

Anaphylaxis is a clinical syndrome characterized by

- sudden onset AND

- rapid progression of signs and symptoms AND

- involving multiple $(\geq 2)$ organ systems, as follows

Level 1 of diagnostic certainty

- $\geq 1$ major dermatological AND

- $\geq 1$ major cardiovascular AND/OR 1 major respiratory criterion

Level 2 of diagnostic certainty

- $\geq 1$ major cardiovascular AND $\geq 1$ major respiratory criterion OR

- $\geq 1$ major cardiovascular OR respiratory criterion AND

- $\geq 1$ minor criterion involving $\geq 1$ different system (other than cardiovascular or respiratory systems) OR

- ( $\geq 1$ major dermatologic) AND ( $\geq 1$ minor cardiovascular AND/OR minor respiratory criterion)

Level 3 of diagnostic certainty

- $\geq 1$ minor cardiovascular OR respiratory criterion AND

- $\geq 1$ minor criterion from each of $\geq 2$ different systems/categories

*The case definition should be applied when there is no clear alternative diagnosis for the reported event to account for the combination of symptoms.

tReprinted, with permission from Elsevier, from Vaccine, Vol 25, Ruggeberg JU, Gold M, Bayas JM, et al; The Brighton Collaboration Anaphylaxis Working Group. ${ }^{2}$ Anaphylaxis: case definition and

guidelines for data collection, analysis, and presentation of

immunization safety data. p. 5675-84. () 2007.

New South Wales, with a population of 6.8 million, accounts for about one-third of the Australian population. School-based vaccination began in April 2007 for those aged 15 and older (enrolled female cohort about 114 000). In order to complete the course by the end of the school year in December, an accelerated vaccination schedule of 0,1 and 4 months was used.

\section{Identification and review of anaphylaxis episodes}

Vaccines were administered by teams of nurses who provided a summary of doses administered and reported any adverse events following immunization daily by fax to the New South Wales Health Immunization unit. Notification of an adverse event, defined as an unwanted or unexpected event after the administration of vaccine, is mandatory under the New South Wales Public Health Act. Nationally, all such notifications are forwarded to the Adverse Drug Reactions Advisory Committee, a subcommittee of Australia's drug and therapeutics regulatory authority, the Therapeutic Goods Administration.

A panel of 13 people with a range of expertise (program delivery, pediatric allergy, surveillance of adverse events after vaccination and public health) was convened to review reports of adverse events following HPV vaccination. To determine the rates of anaphylaxis, we reviewed all reports of 1 or more of skin rash within 48 hours of immunization $(n=40)$, presumptive anaphylaxis $(n=8)$ or allergy $(n=28)$, and any cases where adrenaline had been administered $(n=9)$ or with respiratory or cardiovascular symptoms or both $(n=16)$.

\section{Comparison with meningococcal vaccination program}

A comparable expert panel had been previously convened to review reports of adverse events following a school-based meningococcal C vaccination program in 2003. This program, part of a national campaign to avert a further increase in cases of meningococcal $\mathrm{C}$ disease in Australia, administered 823197 doses of vaccine to boys and girls aged 5-19 years. This panel classified relevant adverse events as anaphylaxis $(n=1)$, allergic reaction $(n=13)$ or rash $(n=10)$; there were 4 children who received adrenaline (New South Wales Health, unpublished data). We compared the rates of suspected anaphylaxis in this program to those in the HPV program, given similar surveillance systems and teams of school-based vaccinators. Similarly, in 1998 a national campaign to prevent a measles epidemic, with a large schoolbased component, delivered 1.7 million doses of measles-mumps-rubella vaccine to Australian children aged 5-12 years. An adverse events panel conducted an investigation into reports of allergic reactions. ${ }^{5}$

\section{Clinical evaluation}

Following an initial review, we contacted patients suspected to have had anaphylaxis, or their guardians, for written consent to conduct a telephone interview and to access their medical records (ambulance and hospital). Trained staff used a standardized, piloted questionnaire developed for this study for telephone interviews with the patient, the patient's guardian and the immunization provider who witnessed or managed the reaction. The patient and guardian questionnaire included demographic information, past medical history (especially of atopic disease and allergies), presenting symptoms, treatment and outcome, and immunization history. The provider questionnaire collected details of the preimmunization assessment of the patient, history of latex exposure, symptoms reported at the time of the event, clinical signs, treatment and overall clinical impression.

We invited all patients with suspected anaphylaxis to attend The Children's Hospital at Westmead for allergy testing at least 6 weeks after the episode. The allergy testing protocol included skin-prick testing for a panel of common antigens (cat, rye grass pollen, alternaria, house dust mite), vaccine components (baker's yeast [Saccharomyces cerevisiae], polysorbate 80) and the HPV vaccines Gardasil and Cervarix (GlaxoSmithKline). We performed intradermal testing (0.02 mL per injection) with HPV vaccines and polysorbate 80 (dilutions 1:100 and 1:10). The vaccine adjuvant (proprietary Merck amorphous aluminum hydroxyphosphate sulfate) was not available for inclusion in the protocol.

Following investigation, we classified cases using the Brighton case definition of anaphylaxis and the level of diagnostic certainty ${ }^{2}$ (Box 1, Box 2). The Brighton definition refers to the level of diagnostic certainty but not the degree of severity of the case (i.e., a level 1 classification is associated with the greatest diagnostic certainty of anaphylaxis). For the purpose of applying the definition, we considered a rash that occurred at any site not contiguous with the injection site to be "generalized." 
We based our causality assessment on the timing of the onset of symptoms, according to the World Health Organization classification (i.e., certain/very likely, probable, possible, unlikely, unrelated), ${ }^{4}$ and whether or not an alternative trigger was present.

\section{Analysis}

We calculated rates using the known number of administered doses from the school-based programs as the denominator and calculated exact Poisson 95\% confidence intervals (CI). We also extracted published rates of anaphylaxis from other population-based vaccination programs and calculated 95\% CIs.

\section{Funding and commercial interests}

This study was funded by the New South Wales Department of Health. The manufacturers of the HPV vaccine did not provide funding for this study.

\section{Results}

In the 2007 school-based program, there were 269680 doses of HPV vaccine administered. This consisted of dose 1 ( $n=95006,83 \%$ coverage), dose 2 ( $n=91289,80 \%$ coverage) and dose 3 ( $n=83845,74 \%$ coverage). Of 155 adverse events reported after HPV vaccination, 110 were for vaccinations in the school program (41 per 100000 doses). In the general practice program, there were 347568 doses distributed, but the number of doses administered is not known. We initially classified 12 cases as requiring further investigation for suspected anaphylaxis. However, 1 patient did not provide consent for a telephone interview or medical record review, and thus was not further classified. Among the 12 cases, there was no geographic clustering or vaccine batch number implicated.

We reviewed all data for the 11 patients with suspected anaphylaxis, and we classified $8(73 \%)$ as fulfilling the Brighton case definition (Table 1). Of these, 1 case was level 1 , and 7 were level 2 . Because the onset of symptoms occurred within 30 minutes of vaccination and because there was no alternate explanation, we determined that these cases were certain or very likely to have been caused by the vaccine. We considered and rejected the possibility that these cases could have been vasovagal episodes or somatic conversion disorder misdiagnosed as anaphylaxis. Of the 8 cases, 6 had generalized urticaria, 1 had angioedema and 1 had no dermatological signs but had stridor and hypoxia. Of the 3 cases not classified as anaphylaxis, 2 were classified as generalized allergic reactions on the basis of failure to progress, and 1 was considered unlikely to be a case of anaphylaxis because of the absence of objective signs and safe receipt of a subsequent dose. No alternate diagnosis could be suggested based on the available evidence. This person had been receiving high-dose steroids for Crohn disease at the time of the reaction.

Of the 8 confirmed cases, 7 were reported from the schoolbased program. The median age was 16.5 (min-max 15-25) years. Six cases were reported after dose 1 of the vaccine, and

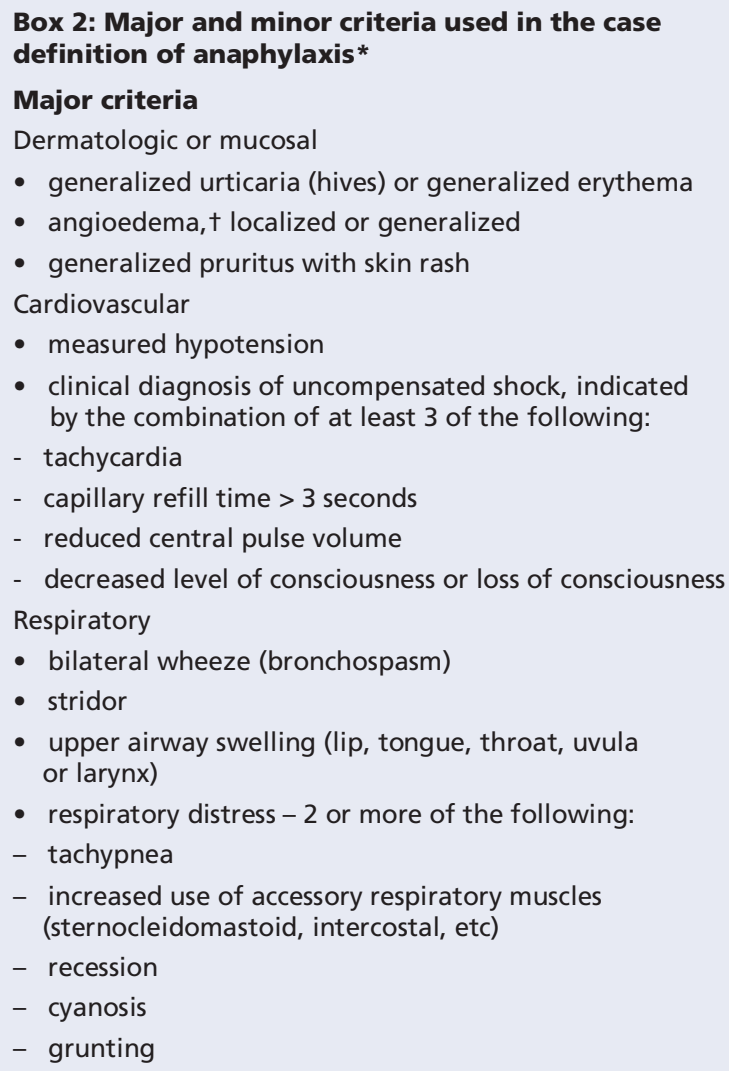

- clinical diagnosis of uncompensated shock, indicated by the combination of at least 3 of the following:

- tachycardia

- capillary refill time $>3$ seconds

- reduced central pulse volume

decreased level of consciousness or loss of consciousness

Respiratory

- bilateral wheeze (bronchospasm)

- stridor

- upper airway swelling (lip, tongue, throat, uvula or larynx)

- respiratory distress -2 or more of the following:

- tachypnea

- increased use of accessory respiratory muscles (sternocleidomastoid, intercostal, etc)

- recession

- cyanosis

- grunting

\section{Minor criteria}

Dermatologic or mucosal

- generalized pruritus without skin rash

- generalized prickle sensation

- localized injection site urticaria

- red and itchy eyes

Cardiovascular

- reduced peripheral circulation as indicated by the combination of at least 2 of

- tachycardia and

- a capillary refill time of $>3$ seconds without hypotension

- a decreased level of consciousness

Respiratory

- persistent dry cough

- hoarse voice

- difficulty breathing without wheeze or stridor

- sensation of throat closure

- sneezing, rhinorrhea

Gastrointestinal

- diarrhea

- abdominal pain

- nausea

- vomiting

Laboratory

- Mast cell tryptase elevation > upper normal limit

*Reprinted, with permission from Elsevier, from Vaccine, Vol 25, Ruggeberg JU, Gold M, Bayas JM, et al; The Brighton Collaboration Anaphylaxis Working Group. ${ }^{2}$ Anaphylaxis: case definition and guidelines for data collection, analysis, and presentation of immunization safety data. p. 567584. ๑ 2007.

tNot hereditary angioedema. 


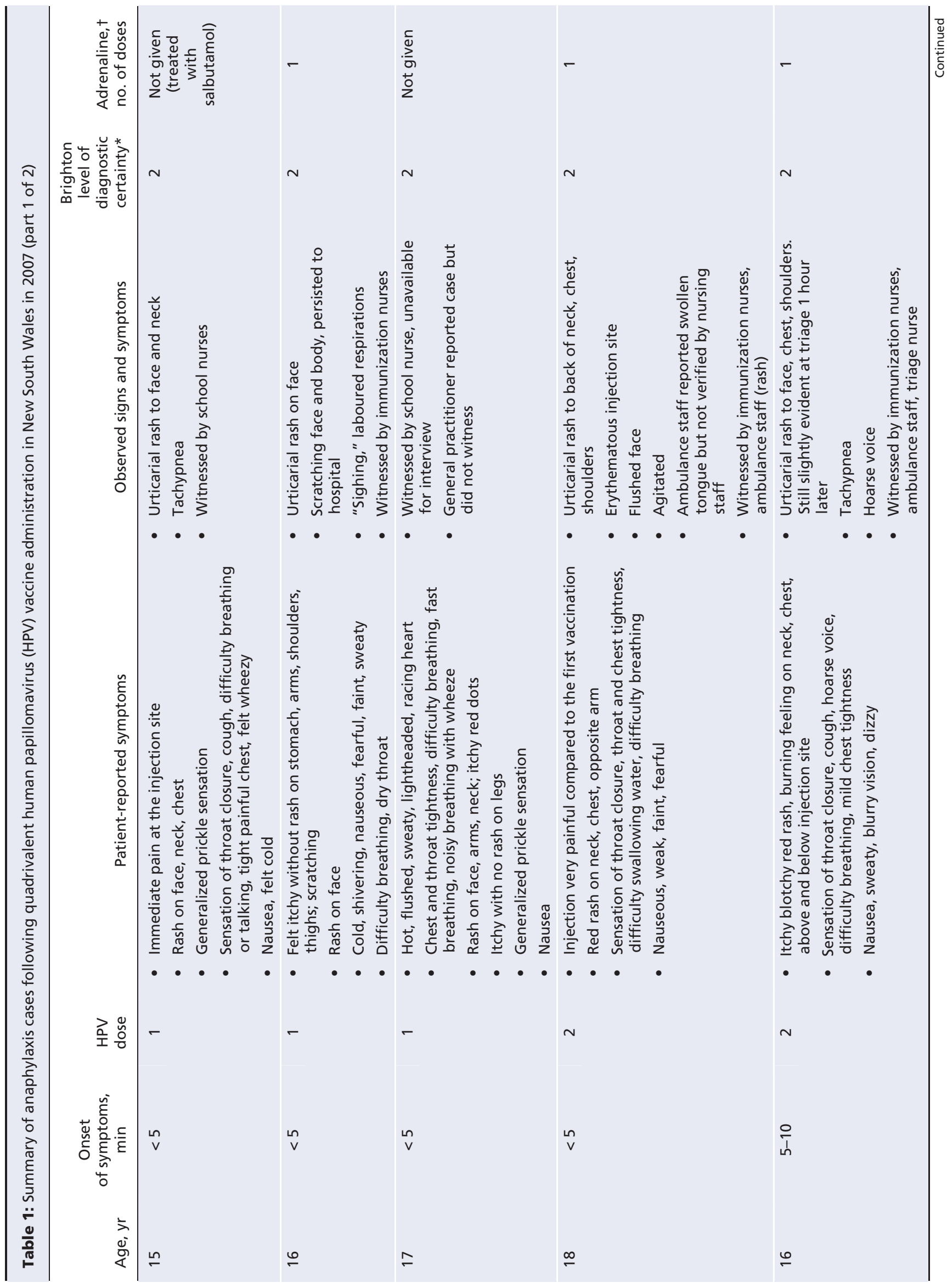




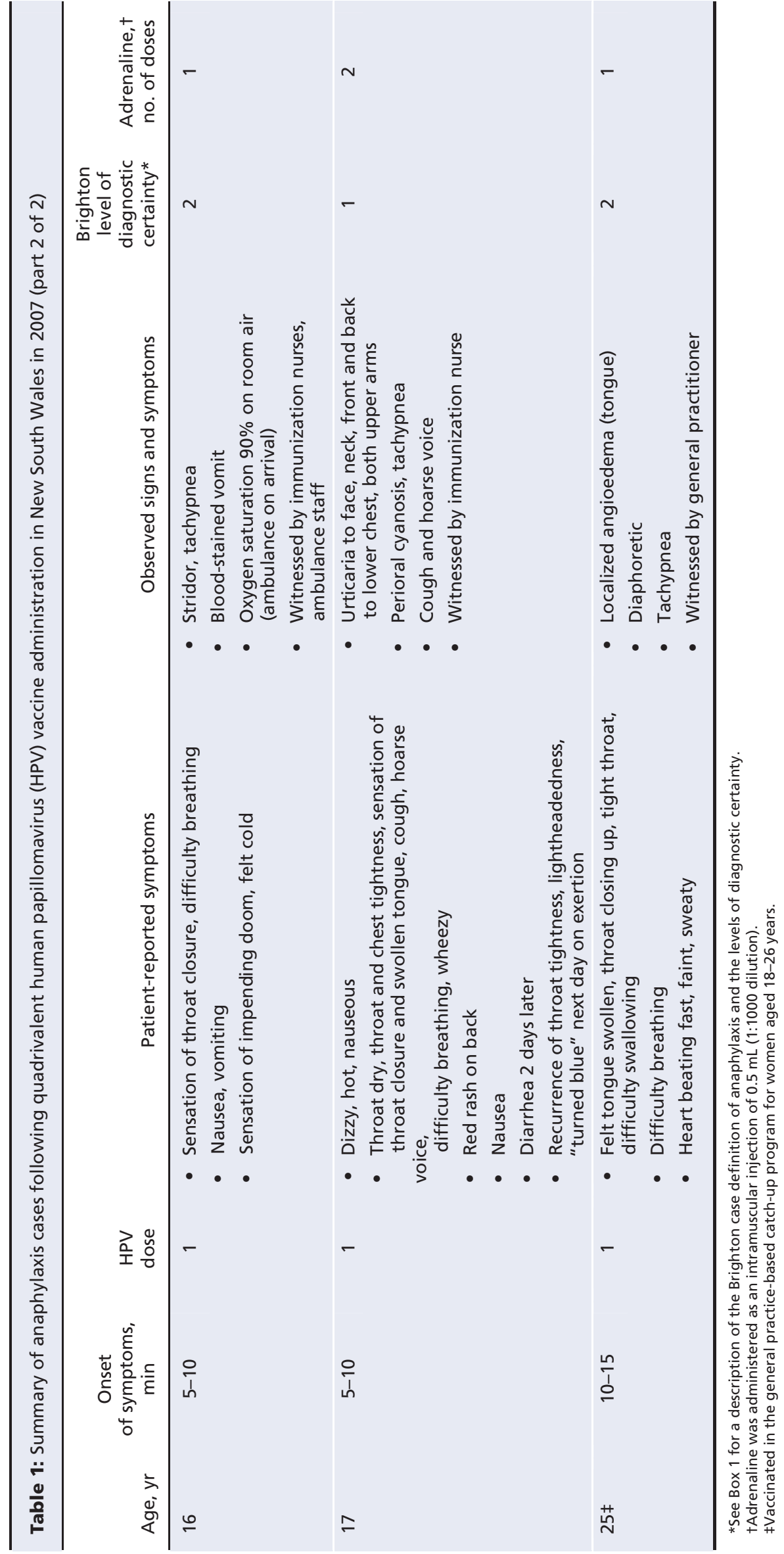

2 cases were reported after dose 2 . Neither of the latter 2 patients had reported a reaction to their first dose of the HPV vaccine. Four patients had a history of allergic reactions and atopic disease (1 had asthma, 3 had eczema or urticaria, 3 had allergic rhinitis). All had been fully vaccinated according to the recommendations for their age group, and anaphylaxis had not developed with previous vaccinations.

Four cases of anaphylaxis (one level 1 case, three level 2 cases) received allergy testing. Of these, 2 had a positive reaction to skin-prick testing for house dust mites. All had negative reactions to skin-prick testing for baker's yeast, and to skin-prick testing and intradermal testing for Gardasil, Cervarix and polysorbate 80 .

Based on the 7 confirmed cases of anaphylaxis from the school-based program (269 680 doses administered), the estimated rate of anaphylaxis following HPV vaccination was 2.6 per 100000 doses administered (95\% CI 1.0-5.3 per 100 000). Five cases occurred after dose 1 of the vaccine (95006 doses administered), giving a rate of anaphylaxis of 5.3 per 100000 people vaccinated (95\% CI 1.7-12.3). Two cases occurred after the second dose (91 289 doses administered), giving a rate of anaphylaxis of 2.2 per 100000 people vaccinated (95\% CI 0.3-7.9).

In comparison, the 2003 schoolbased meningococcal $\mathrm{C}$ vaccination program had 1 identified case of anaphylaxis (anaphylaxis rate 0.1 per 100000 doses administered, 95\% CI 0.003-0.7). A 13-year-old boy, who was coadministered the hepatitis B vaccine, immediately developed tachycardia, diaphoresis, chest pain, an itchy blocked throat, dysphagia, vomiting, agitation and a blotchy rash on his neck. He required 2 doses of adrenaline. On chart review performed for this study, 2 additional cases (girls aged 11 and 12 years) would have been assessed for suspected anaphylaxis in the current investigation, and they may have met the Brighton definition for anaphylaxis if full clinical information were available. One girl had complained of tongue swelling, throat tightness, and chest and abdominal pain 5 minutes after vaccination, and the other reported difficulty breathing and a 
rash on her neck and leg and at the injection site 1.5 hours after vaccination. If we include these cases, the anaphylaxis rate is 0.4 cases per 100000 doses (95\% CI 0.1-1.1).

Rates of anaphylaxis reported from other immunization programs are given in Table 2 and Appendix 1 (www.cmaj.ca /cgi/content/full/179/6/525/DC2).

\section{Interpretation}

The anaphylaxis rate in the 2007 school-based HPV vaccination program was 2.6 per 100000 doses (95\% CI 1.0-5.3 per 100000 doses). This is higher than reported for other vaccines (Table 2). The rate of anaphylaxis after HPV vaccination is comparable only to the rate following administration of vaccines containing bovine gelatin in Japan (Table 2), which led to removal of the gelatin from the vaccine. ${ }^{17}$ The lower limit of the confidence interval for the rate of anaphylaxis in the New South Wales HPV vaccination program overlaps with the upper limit of the confidence interval for the Canadian hepatitis B vaccination program and UK measles-rubella vaccination program (Table 2). Although the point estimate for the rate of anaphylaxis is higher than in previous programs, the estimated rate is still an order of magnitude less common than the World Health Organization categorization of adverse events after immunization which are "very rare" (< 1 in 10000$)^{4}$

Unpublished safety data from the HPV vaccine manufacturer indicate that no vaccine safety signal for allergy or anaphylaxis was identified in the vaccine trials. ${ }^{18}$ However, it is well recognized that prelicensure trials are underpowered to detect anaphylaxis. ${ }^{2}$ Hypersensitivity reactions to HPV vaccine were the fifth most common adverse event reported to the Vaccine Adverse Events Reporting System in 2007, ${ }^{19}$ and skin rash within 48 hours accounted for $26 \%$ of the reports of adverse events after HPV vaccination in New South Wales in 2007.

Reasons for the increased rate of anaphylaxis after HPV vaccine in the New South Wales school-based program are not clear but could be due to a number of factors including possible allergenicity of components of the HPV vaccine, enhanced passive adverse event surveillance and use of the Brighton definition of anaphylaxis. Another potential factor is that, from midadolescence onward, women have higher rates of anaphylaxis than men..$^{20,21}$ An English study found that the risk of hospital admission for anaphylaxis is considerably higher among women of child-bearing age than in the rest of the population (rate ratio $1.3895 \%$ CI $1.27-$ 1.50). ${ }^{21}$ The incidence of anaphylaxis in Australia also appears to be increasing. ${ }^{20} \mathrm{~A}$ recent analysis by Siegrist and

Table 2: Summary of rates of anaphylaxis following immunization

\begin{tabular}{|c|c|c|c|c|}
\hline Surveillance type, program & Period & Vaccine & $\begin{array}{l}\text { Anaphylaxis rate, } \\
\text { per } 100000\end{array}$ & $\begin{array}{l}95 \% \text { Poisson } \\
\text { confidence interval }\end{array}$ \\
\hline \multicolumn{5}{|l|}{ School-based program } \\
\hline New South Wales & Apr-Dec 2007 & Quadrivalent HPV & 2.60 & $1.04-5.35$ \\
\hline D Souza et al. ${ }^{5}$ & Aug-Nov 1998 & Measles-mumps-rubella & 0.41 & $0.17-0.85$ \\
\hline $\begin{array}{l}\text { New South Wales Department of } \\
\text { Health }\end{array}$ & 2003 & Conjugated meningococcal C & 0.12 & $0.003-0.68$ \\
\hline Dobson et al. ${ }^{6}$ & 1992 & Hepatitis B & 0.78 & $0.02-4.36$ \\
\hline UK Department of Health ${ }^{7}$ & 1994 & Measles-rubella & 1.00 & $0.79-1.24$ \\
\hline \multicolumn{5}{|l|}{ Enhanced surveillance } \\
\hline \multirow[t]{5}{*}{ Sakaguchi et al. ${ }^{8}$} & \multirow[t]{5}{*}{ Apr 1994-Mar 1997} & Measles & 1.68 & $1.28-2.17$ \\
\hline & & Mumps & 1.96 & $0.90-3.72$ \\
\hline & & Rubella & 1.60 & $1.02-2.37$ \\
\hline & & Varicella & 2.35 & $1.35-3.82$ \\
\hline & & Varicella 1996 (peak rate) & 4.52 & $2.33-7.89$ \\
\hline Patja et al. ${ }^{9,10}$ & 1982-1996 & Measles-mumps-rubella & 0.05 & $0.02-0.08$ \\
\hline \multirow[t]{2}{*}{ Bohlke et al. ${ }^{11}$} & \multirow[t]{2}{*}{ 1991-1997 } & All vaccines, all study sites & 0.07 & $0.02-0.15$ \\
\hline & & All vaccines, enhanced site & 0.15 & $0.004-0.85$ \\
\hline \multicolumn{5}{|l|}{ Passive national surveillance } \\
\hline \multirow[t]{3}{*}{ Nakayama and Onoda ${ }^{12}$} & \multirow[t]{3}{*}{ 1994-2004 } & Japanese encephalitis & 0.06 & $0.02-0.14$ \\
\hline & & $\begin{array}{l}\text { Diphtheria-tetanus-pertussis } \\
\text { (acellular) }\end{array}$ & 0.09 & $0.05-0.17$ \\
\hline & & Influenza & 0.07 & $0.04-0.10$ \\
\hline Pool et al. ${ }^{13}$ & 1991-1997 & Measles-mumps-rubella & 0.18 & $0.15-0.21$ \\
\hline UK Department of Health ${ }^{14}$ & 1997-2003 & All vaccines & 0.11 & $0.09-0.13$ \\
\hline Zhou et al. ${ }^{15}$ & $1991-2001$ & All vaccines & 0.02 & $0.022-0.026$ \\
\hline Kelso et al. ${ }^{16}$ & 1990-1997 & Yellow fever & 0.76 & $0.55-1.04$ \\
\hline
\end{tabular}


colleagues $^{22}$ anticipated the identification of vaccine safety signals for allergic and autoimmune diseases following population implementation of HPV vaccination because medical presentations for allergy-related conditions in young women are common. It is therefore important when assessing causality to note that all of our cases occurred within minutes of vaccination.

The school-based program in New South Wales might be expected to have high sensitivity for adverse events occurring soon after vaccination because experienced nursing teams provide both vaccine and postvaccination observation. However, the same arrangements were in place for the recent school-based vaccination program delivering meningococcal $\mathrm{C}$ vaccines to a similar age group in this region and no such signal was detected. Present definitions of anaphylaxis reflect the understanding that it has a variable spectrum of severity and presenting symptoms. This is an important issue when comparing rates with previous published reports, which are likely to be restricted to the most severe cases only. However, when we applied the definitions used in other recent studies of anaphylaxis following immunization to our cases, they all satisfied the case definition for probable anaphylaxis..$^{11,13,23} \mathrm{We}$ found the Brighton classification useful, but we considered it inappropriate for screening because routine surveillance reports of adverse events following vaccination usually lack the necessary level of clinical detail.

Pre-existing allergen sensitization is biologically plausible for several components of the quadrivalent HPV vaccine. Natural HPV infection does not cause viremia; however, HPV virus-like particles are highly immunogenic when injected. ${ }^{24}$ It is unclear whether some people may produce an $\mathrm{IgE}$ response to injected HPV antigens. One small trial injected participants $(n=30)$ with HPV type 11 virus-like particles and found no increase in IgE levels ${ }^{25}$ however, data from a study of rhesus macaques $(n=10)$ suggests it is possible. ${ }^{26}$ Because the age group targeted by the HPV vaccination program was 15 years and older, many women may have already been exposed to HPV, because the median age of first sexual intercourse in Australia is 16 years. ${ }^{27}$ There is potential for residual amounts of yeast proteins to be in the HPV vaccine. Although hypersensitivity to yeast is a documented phenomenon, ${ }^{28}$ there have been infrequent reports of anaphylaxis or hypersensitivity reactions following the receipt of yeastcontaining vaccines..$^{29-32}$

Quadrivalent HPV vaccine contains polysorbate 80 as a stabilizer. ${ }^{33}$ Polysorbates are commonly used as solubilizers, stabilizers and emulsifiers in cosmetics and medical preparations. Polysorbate 80 could be a potential trigger of nonallergic ("anaphylactoid") anaphylaxis. There have been a number of reports of anaphylaxis or generalized hypersensitivity reactions occurring following the receipt of drugs that have implicated polysorbate 80 as the cause. ${ }^{34-37}$ Aluminum adjuvants are not a documented cause of anaphylaxis. ${ }^{38}$

The most common event that may be confused with anaphylaxis during an acute episode is syncope. Syncope has also been reported following HPV vaccination, ${ }^{19}$ but its onset is typically more rapid, with a person becoming unconscious in seconds. ${ }^{39}$ The absence of urticaria, pruritus, angioedema or upper respiratory obstruction are helpful in differentiating syncope from anaphylaxis, as are the presence of pallor instead of flushing and nausea without abdominal pain. ${ }^{40}$

All of the people with anaphylaxis in the New South Wales HPV vaccination program recovered completely. Most did so rapidly after administration of adrenaline and were asymptomatic by the time they reached the emergency department (20-60 minutes later). In such cases, primary care staff may need to rely on witness and patient history to obtain a complete clinical picture.

In July 2007, the package insert for the quadrivalent HPV vaccine was updated to reflect reports of anaphylaxis following HPV vaccination, and the product information has been updated internationally by the manufacturer. Following confirmation of the vaccine safety signal, all general practitioners in New South Wales were reminded via fax to be prepared for the occurrence of anaphylaxis following HPV vaccination and to have appropriate protocols and equipment available. Consent forms used in the schoolbased program include the small but real risk of anaphylactic reaction. In December 2007, the Therapeutic Goods Administration placed advice on its website alerting providers to the occurrence of anaphylaxis following HPV vaccination. ${ }^{41}$ In 2008, the New South Wales school-based HPV vaccination program is targeted to girls aged $12-15$ years. As of May 2008, 181235 doses of HPV vaccine have been administered and 78 adverse events following vaccination have been reported. Twenty-two cases of "allergic reaction" have been reported, but no reports of anaphylaxis have been received.

\section{Limitations}

This study had several limitations. First, because expert bodies find it difficult to define anaphylaxis, the classification of a case as anaphylaxis will be somewhat imprecise and dependent on the particular definition used. The available details are those recorded by the immunization nurses and are not as complete as those documented in a clinical trial setting. Full clinical details were not available for all cases. Second, there was a delay of weeks to months between the event and the interview, because of delays in reporting, obtaining consent and availability of cases for interview. Third, the small number of cases identified in this and other reports of anaphylaxis following vaccination makes estimates of incidence imprecise and comparison between case series vulnerable to misclassification. This makes the true magnitude of the difference between the incidence identified here and that for other vaccines uncertain, with misclassification of only 1 or 2 cases capable of substantially altering estimates of fold differences. This is especially true when differences in the propensity for the occurrence and recognition of possible anaphylaxis by age group and sex are taken into account. ${ }^{2,20-22}$ Nevertheless, we attempted to be exhaustive in our evaluation of the cases, and the case identification pathways were identical to those in recent comparable school-based campaigns. School-based HPV vaccination campaigns are being conducted in Canada, the United Kingdom and elsewhere, so awareness of this experience should focus attention on the 
identification of anaphylaxis and lesser possible allergic manifestations, such as urticarial rash, in order to enlarge the available denominator

\section{Conclusion}

Anaphylaxis following HPV vaccination is a rare event, as defined by the World Health Organization, ${ }^{4}$ and it should not curtail population-based HPV vaccination programs. It is noteworthy that no cases of anaphylactic shock occurred, but it is important to note the wide clinical spectrum of anaphylaxis. ${ }^{2}$ Although this makes confirming that these cases truly represent anaphylaxis difficult, it also highlights the importance of good training for staff administering vaccines in school or other settings in the recognition and management of suspected anaphylaxis and its reporting. Routine reporting forms for adverse events following vaccination could include prompts to ensure that enough detail is recorded to allow subsequent classification using the Brighton definition of anaphylaxis. The biological basis for the reported reactions to HPV vaccine requires further research.

\section{This article has been peer reviewed.}

Competing interests: None declared for Mike Gold and Sue CampbellLloyd. Julia Brotherton and Peter McIntyre received partial grant funding from Commonwealth Serum Laboratories and GlaxoSmithKline for an investigator-driven study of HPV prevalence in Australia. They also received funding for the laboratory testing component of a serosurvey of HPV antibodies in Australia from Commonwealth Serum Laboratories. Andrew Kemp owns shares of Commonwealth Serum Laboratories and is a member of the Asia-Pacific Immunoglobulins in Immunology Expert group. His travel to meetings for this group has been supported by Commonwealth Serum Laboratories. Margaret Burgess received travel assistance from GlaxoSmithKline to attend the 2007 European Society for Paediatric Infectious Diseases meeting.

Contributors: All of the authors contributed to the study design. Julia Brotherton developed the study protocol and designed the study questionnaires. Mike Gold, Andrew Kemp, Peter McIntyre and Margaret Burgess reviewed the study questionnaires. Julia Brotherton coordinated data entry and managed the study team. Mike Gold developed the allergy testing protocol. Andrew Kemp supervised the allergy testing. Sue Campbell-Lloyd managed the study, including the consent process and funding. Julia Brotherton performed the data analysis and, along with Mike Gold, Andrew Kemp, Peter McIntyre and Margaret Burgess, interpreted the data. All of the authors revised the manuscript for important intellectual content and approved the final version submitted for publication.

Acknowledgements: We thank the staff of the National Centre for Immunisation Research and Surveillance who assisted with the implementation of this study: Dr. Nicholas Wood, Ms. Pamela Cheung, Ms. Margaret Pym, Ms. Rosemary Joyce, Ms. Kylee Gibbins, Dr. Jane Jelfs, Ms. Catherine King, Ms. Donna Armstrong, Ms. Kirsty Whybrow and Ms. Lynda Beaumont. We also thank Dr. Miriam Codarini who assisted with interviewing and clinical review, and Dr. Shelley Deeks for helpful comments on the manuscript.

The National Centre for Immunisation Research and Surveillance is supported by the Australian Government Department of Health and Ageing, the New South Wales Department of Health and The Children's Hospital at Westmead.

Funding: This study was funded by the New South Wales Department of Health.

\section{REFERENCES}

1. Babl FE, Lewena S, Brown L. Vaccination-related adverse events. Pediatr Emerg Care 2006;22:514-9.

2. Rüggeberg JU, Gold MS, Bayas JM, et al. The Brighton Collaboration Anaphylaxis Working Group. Anaphylaxis: case definition and guidelines for data collection, analysis, and presentation of immunization safety data. Vaccine 2007;25:5675-84.
3. Sampson HA, Munoz-Furlong A, Campbell RL, et al. Second symposium on the definition and management of anaphylaxis: summary report-Second National Institute of Allergy and Infectious Disease/Food Allergy and Anaphylaxis Network symposium. J Allergy Clin Immunol 2006;117:391-7.

4. World Health Organization. Adverse events following immunization: causality assessment. Geneva: The Organization; 2008. Available: www.who.int/vaccinesdocuments/DocsPDF05/815.pdf (accessed 2008 July 21).

5. D'Souza RM, Campbell-Lloyd S, Isaacs D, et al. Adverse events following immunisation associated with the 1998 Australian Measles Control Campaign. Commun Dis Intell 2000;24:27-33.

6. Dobson S, Scheifele D, Bell A. Assessment of a universal, school-based hepatitis B vaccination program. JAMA 1995;274:1209-13.

7. Salisbury DM, Campbell H, Edwards B. Measles rubella immunisation campaign in England "one year on." London (UK): Department of Health; 1995.

8. Sakaguchi M, Nakayama T, Fujita H, et al. Minimum estimated incidence in Japan of anaphylaxis to live virus vaccines including gelatin. Vaccine 2000;19:431-6.

9. Patja A, Davidkin I, Kurki T, et al. Serious adverse events after measlesmumps-rubella vaccination during a fourteen-year prospective follow-up. Pediatr Infect Dis J 2000;19:1127-34.

10. Patja A, Mäkinen-Kiljunen S, Davidkin I, et al. Allergic reactions to measles-mumps-rubella vaccination. Pediatrics 2001;107:e27.

11. Bohlke K, Davis RL, Marcy SM, et al. Risk of anaphylaxis after vaccination of children and adolescents. Pediatrics 2003;112:815-20.

12. Nakayama T, Onoda K. Vaccine adverse events reported in post-marketing study of the Kitasato Institute from 1994 to 2004. Vaccine 2007;25:570-6.

13. Pool V, Braun MM, Kelso JM, et al. Prevalence of anti-gelatin IgE antibodies in people with anaphylaxis after measles-mumps rubella vaccine in the United States. Pediatrics 2002;110:e71.

14. Department of Health. Vaccine safety and the management of adverse events following immunisation. In: Salisbury D, Ramsay M, Noakes K, eds. Immunisation against infectious disease: "The Green Book." London (UK): The Stationery Office; 2006. p. 53-64. Available: www.dh.gov.uk/en/Publichealth/Healthprotection /Immunisation/Greenbook/DH_4097254 (accessed 2008 May 23).

15. Zhou W, Pool V, Iskander JK, et al. Surveillance for safety after immunization: Vaccine Adverse Event Reporting System (VAERS) — United States, 1991-2001. MMWR Surveill Summ 2003;52:1-24

16. Kelso JM, Mootrey GT, Tsai TF. Anaphylaxis from yellow fever vaccine. $J$ Allergy Clin Immunol 1999;103:698-701.

17. Kuno-Sakai H, Kimura M. Removal of gelatin from live vaccines and DTaP-an ultimate solution for vaccine-related gelatin allergy. Biologicals 2003;31:245-9.

18. Miller NB, US Food and Drug Authority. Clinical review of Biologics License Application for Human papillomavirus $6,11,16,18 \mathrm{~L} 1$ virus like particle vaccine $(S$. cerevisiae) (STN 125126 GARDASIL), manufactured by Merck, Inc. 2006. Available: www.fda.gov/cber/review/hpvmer060806r.pdf (accessed 2008 July 21).

19. Huang WT, Leidel L, Vellozi C, et al. Safety review of quadrivalent human papillomavirus (types $6,11,16,18$ ) recombinant vaccine [poster abstract 150]. International Conference on Emerging Infectious Diseases 2008; Mar. 16-19; Atlanta (GA). Available: www.cdc.gov/eid/content/14/3/ICEID2008.pdf (accessed 2008 July 21).

20. Poulos LM, Waters AM, Correll PK, et al. Trends in hospitalizations for anaphylaxis, angioedema, and urticaria in Australia, 1993-1994 to 2004-2005. J Allergy Clin Immunol 2007;120:878-84.

21. Sheikh A, Alves B. Age, sex, geographical and socio-economic variations in admissions for anaphylaxis: analysis of four years of English hospital data. Clin Exp Allergy 2001;31:1571-6.

22. Siegrist CA, Lewis EM, Eskola J, et al. Human papilloma virus immunization in adolescent and young adults: a cohort study to illustrate what events might be mistaken for adverse reactions. Pediatr Infect Dis J 2007;26:979-84.

23. DiMiceli L, Pool V, Kelso JM, et al. Vaccination of yeast sensitive individuals: review of safety data in the US vaccine adverse event reporting system (VAERS) Vaccine 2006;24:703-7.

24. Stanley M, Lowy DR, Frazer I. Chapter 12: Prophylactic HPV vaccines: underlying mechanisms. Vaccine 2006;24(Suppl 3):S106-13.

25. Emeny RT, Wheeler CM, Jansen KU, et al. Priming of human papillomavirus type 11 -specific humoral and cellular immune responses in college-aged women with a virus-like particle vaccine. J Virol 2002;76:7832-42.

26. Ruiz W, McClements WL, Jansen KU, et al. Kinetics and isotype profile of antibody responses in rhesus macaques induced following vaccination with HPV 6, 11,16 and $18 \mathrm{~L} 1$-virus-like particles formulated with or without Merck aluminium adjuvant. J Immune Based Ther Vaccines 2005;3:1-11.

27. Rissel CE, Richters J, Grulich AE, et al. Sex in Australia: selected characteristics of regular sexual relationships. Aust N Z J Public Health 2003;27:124-30.

28. Airola K, Petman L, Makinen-Kiljunen S. Clustered sensitivity to fungi: anaphylactic reactions caused by ingestive allergy to yeasts. Ann Allergy Asthma Immunol 2006;97:294-7.

29. Systemic adverse effects of hepatitis B vaccines are rare. Prescrire Int 2004;13: 218-23

30. Brightman CA, Scadding GK, Dumbreck LA, et al. Yeast-derived hepatitis B vaccine and yeast sensitivity. Lancet 1989;333:903.

31. Hudson TJ, Newkirk M, Gervais F, et al. Adverse reaction to the recombinant hepatitis B vaccine. J Allergy Clin Immunol 1991;88:821-2.

32. Barbaud A, Trechot P, Reichert-Penetrat S, et al. Allergic mechanisms and urticaria/angioedema after hepatitis B immunization. Br J Dermatol 1998;139:925-6.

33. Bryan JT. Developing an HPV vaccine to prevent cervical cancer and genital 
warts. Vaccine 2007;25:3001-6.

34. Grims RH, Kranke B, Aberer W. Pitfalls in drug allergy skin testing: false-positive reactions due to (hidden) additives. Contact Dermatitis 2006;54:290-4.

35. ten Tije AJ, Verweij J, Loos WJ, et al. Pharmacological effects of formulation vehicles: implications for cancer chemotherapy. Clin Pharmacokinet 2003;42:665-85.

36. Steele RH, Limaye S, Cleland B, et al. Hypersensitivity reactions to the polysorbate contained in recombinant erythropoietin and darbepoietin. Nephrology 2005; $10: 317-20$

37. Coors EA, Seybold H, Merk HF, et al. Polysorbate 80 in medical products and nonimmunologic anaphylactoid reactions. Ann Allergy Asthma Immunol 2005;95:593-9.

38. Jefferson T, Rudin M, Di Pietrantonj C. Adverse events after immunisation with aluminium-containing DTP vaccines: systematic review of the evidence. Lancet Infect Dis 2004;4:84-90.

39. Braun MM, Patriarca PA, Ellenberg SS. Syncope after immunization. Arch Pediatr Adolesc Med 1997;151:255-9.

40. Walker S, Sheikh A. Managing anaphylaxis: effective emergency and long-term care are necessary. Clin Exp Allergy 2003;33:1015-8.

41. Therapeutic Goods Administration. Human papillomavirus vaccine (Gardasil). Advice from the Therapeutic Goods Administration. Australia: The Administration; 2008. Available: www.tga.gov.au/alerts/medicines/gardasil.htm (accessed 2008 Aug 6).

Correspondence to: Dr. Julia Brotherton, National Centre for Immunisation Research and Surveillance of Vaccine Preventable Diseases, The Children's Hospital at Westmead, Locked Bag 4001, Westmead, New South Wales 2145, Australia; fax 6129845 1418; juliab2@chw.edu.au

Members of the New South Wales HPV Adverse Event Review Panel: Dr. Kerry Chant (Director, Health Protection, and Deputy Chief Health Officer, New South Wales Department of Health); Ms. Deborah Thomson (Senior Policy Officer, New South Wales Department of Health); Dr. Julia Brotherton (Senior Research Fellow, National Centre for Immunisation Research and Surveillance); Dr. Margaret Burgess (Former Director, National Centre for Immunisation Research and Surveillance); Ms. Sue CampbellLloyd (Manager, Immunisation Unit, New South Wales Department of Health); Dr. Mike Gold (Allergist, South Australian Immunisation Coordination Unit, Australian Drug Reactions Advisory Committee, Therapeutic Goods Administration representative); Dr. Andrew Kemp (Professor of Pediatric Allergy and Clinical Immunology, The Children's Hospital at Westmead); Dr. Glenda Lawrence (Senior Research Fellow, National Centre for Immunisation Research and Surveillance); Dr. Peter McIntyre (Director, National Centre for Immunisation Research and Surveillance); Dr. Conrad Moreira (Director, Communicable Diseases and Immunisation, Sydney West Centre for Population Health); Ms. Sonya Nicholl (Senior Policy Officer, New South Wales Department of Health); Mr. Darryl O'Donnell (Acting Associate Director, AIDS and Infectious Diseases Branch, New South Wales Department of Health); and Dr. Peter Procopis (Pediatric Neurologist, The Children's Hospital at Westmead).

Panel Observers: Ms. Kirsty Whybrow (Administrative officer, National Centre for Immunisation Research and Surveillance); $M r$. Joel Willis (Australian Government Department of Health and Ageing, Immunisation Programs Section); and Dr. Shelley Deeks (Deputy Director of Surveillance, National Centre for Immunisation Research and Surveillance). 